International Journal of Pure and Applied Mathematics

Volume 102 No. 3 2015, 407-419

ISSN: 1311-8080 (printed version); ISSN: 1314-3395 (on-line version)

url: http://www.ijpam.eu

doi: http://dx.doi.org/10.12732/ijpam.v102i3.1



\title{
ASYMPTOTIC LAPLACE TRANSFORMS AND WATSON'S LEMMA
}

\author{
Claudiu Mihai \\ Department of Mathematics \\ Daemen College \\ Amherst, 4380 Main St, Amherst, NY, 14226, USA
}

\begin{abstract}
In this paper we will present yet another definition of the Asymptotic Laplace Transforms which comes to further improve the extension of the Laplace Transforms to all locally integrable function as proposed by G. Lumer and F. Neubrander [7]. We will present two new results, one due essentially to the late G. Lumer, results related to the asymptotic expansion of the Laplace Transforms in terms of $\frac{1}{\lambda}$.
\end{abstract}

\section{Introduction and Preliminary Results}

The concept of asymptotic Laplace transforms has its roots in the theory of asymptotic power series of analytic functions; see, e.g., R. Remmert [10]. Asymptotic Laplace transforms were considered first by J.C. Vignaux [11] and further investigated by J.C Vignaux and M. Cotlar [12], W.A. Ditkin [5], L. Berg [2], Yu. I. Lyubich [9] and M. Deakin [4]. Since slightly less transparent definitions were used, the method and the scope of its applicability remained largely unnoticed. In 1999, G. Lumer and F. Neubrander [7] revisited the asymptotic Laplace transform and applied the concept to verify general theorems for distributions and hyperfunctions semigroups. Although the Lumer-Neubrander definition of the asymptotic Laplace transform was superior to earlier versions,

Received: September 12, 2014

(c) 2015 Academic Publications, Ltd. url: www.acadpubl.eu 
it still had the disadvantage that the operational property $\hat{f}^{\prime}(\lambda)=(\widehat{-t f(t)}) \mathrm{did}$ not extend to the asymptotic setting. To remedy this defect, G. Lumer and F. Neubrander gave yet another definition of the Asymptotic Laplace transform in [8].In this paper we propose a third definition of the asymptotic Laplace transform. Our definition is located somewhere "in-between" the two definitions proposed by G. Lumer and F. Neubrander, and enjoys the good properties of both: i.e., all operational Laplace transform properties are valid and Laplace transforms are "easily computed". As one can see in [1], a function $f \in L_{l o c}^{1}([0, \infty) ; X)$ is Laplace transformable if and only if its antiderivative $F: t \mapsto \int_{0}^{t} f(s) d s$ is exponentially bounded. In this case the Laplace transform $\hat{f}(\lambda)=\int_{0}^{\infty} e^{-\lambda t} f(t) d t=\lambda \int_{0}^{\infty} e^{-\lambda t} F(t) d t$ is analytic and bounded in a right half plane.

Definition 1. We say that $a \approx_{T} 0$ if $a$ is of exponential decay $T$; if

$$
\limsup _{\lambda \rightarrow \infty} \frac{1}{\lambda} \ln \| a(\lambda \| \leq-T
$$

Our new definition of the Asymptotic Laplace transform is based on our observation that the use of postsectors by G. Lumer and F. Neubrander is not necessary in order for the operational properties of the clasical Lapalce transform to remain valid. To state our definition we need the following notation. Denote by $S_{\phi}$ an open cut sector on the right half plane; i.e.,

$$
S_{\phi}:=\left\{\lambda: \lambda=r e^{i \theta}, \quad r>r_{0},|\theta|<\phi<\frac{\pi}{2}\right\} .
$$

We say that an analytic function $u: S_{\phi} \rightarrow X$ is of minimal exponential type on $S_{\phi}$ if for all $\omega>0$ there exists $M>0$ such that $\|u(\lambda)\| \leq M e^{\omega|\lambda|}$ in $S_{\phi}$. We denote by $O_{S_{\phi}}(X)$ the set of $X$-valued analytic functions of minimal exponential type on a given open cut sector $S_{\phi}$. Also, we denote by $O(S, X):=$ $\bigcup_{S_{\phi}} O_{S_{\phi}}(X)$ the set of analytic functions that are of minimal exponential type on some open cut sector $S_{\phi}$. Again, $O(S, X)$ and $O_{S_{\phi}}(X)$ are closed under addition and scalar multiplication; i.e., if $u_{1} \in O_{S_{1}}(X)$ and $u_{2} \in O_{S_{2}}(X)$ then $u_{3}=u_{1}+u_{2} \in O_{S_{3}}(X)$ and $u_{3}=u_{1} \cdot u_{2} \in O_{S_{3}}(X)$ where $S_{3}=S_{1} \cap S_{2}$.

Definition 2. For $0<T<\infty$, the T-asymptotic Laplace transform (of type three) of $f \in L_{l o c}^{1}([0, \infty), X)$ is given by the set of analytic functions $r$ defined on some sectorial region $S$ with values in $X$ which are of the minimal exponential type and are asymptotically equal to the finite Laplace transform of $f$; i.e.,

$$
\{f\}_{3}^{T}:=\left\{r \in O(S, X): r(\lambda)-\int_{0}^{T} e^{-\lambda t} f(t) d t \approx_{T} 0\right\}
$$




$$
=\int_{0}^{T} e^{-\lambda t} f(t) d t+\{0\}_{3}^{T},
$$

where $\{0\}_{3}^{T}=\left\{a \in O(S, X)\right.$ such that $\left.a \approx_{T} 0\right\}$. The asymptotic Laplace transform (of type three) is defined as the intersection of the sets $\{f\}_{3}^{T}$; i.e.,

$$
\begin{aligned}
\{f\}_{3} & :=\cap_{T>0}\{f\}_{3}^{T} \\
& =\left\{r \in O(S, X): r(\lambda)-\int_{0}^{T} e^{-\lambda t} f(t) d t \approx_{T} 0 \text { for all } T>0\right\} .
\end{aligned}
$$

Since the functions of minimal exponential type play an important role in what follows, we give some examples of such functions. For a complete prof on why these functions are of minimal exponential type one can see [3].

Example 3. (a) The functions

$$
u(\lambda)=\lambda^{n} e^{c \lambda^{\gamma}}
$$

are of minimal exponential type in any postsector contained in the region $\{\lambda$ : $|\lambda|>1\} \cap\{\lambda: \operatorname{Re} \lambda>0\}$ for any $n \in \mathbb{R}, \gamma \in[-1,1)$ and $c \in \mathbb{R}$. Notice that $u$ is also of minimal exponential type for $\gamma=1$ provided that $c \leq 0$.

(b) The function

$$
u(\lambda):=\ln (\lambda):=\ln |\lambda|+\operatorname{iarg}(\lambda)
$$

with $-\pi<\arg (\lambda)<\pi$ (the principal branch of the logarithm) is of minimal exponential type in any postsector contained in the region $\{\lambda:|\lambda|>1\} \cap\{\lambda$ : $\operatorname{Re} \lambda>0\}$.

(c) The function

$$
u(\lambda):=e^{-\lambda \ln \lambda}
$$

is of minimal exponential type in any postsector contained in the region $\{\lambda$ : $|\lambda|>1\} \cap\{\lambda: \operatorname{Re} \lambda>0\}$.

(d) The function

$$
u(\lambda):=e^{-\lambda^{2}}
$$

is of minimal exponential type on the sector $S_{\frac{\pi}{4}}:=\left\{\lambda: \lambda=r e^{i \theta}, \quad|\theta|<\frac{\pi}{4}\right\}$, but is not of minimal exponential type on any postsector $\Sigma$.

The following lemma states that if the classical Laplace transform exist then is a member of the asymptotic Laplace transform. For complete proofs of these preliminary results see [3]. 
Lemma 4. Let $a \in O\left(S_{\phi}, X\right)$. The following are equivalent

(a) $a \approx_{T}$ 0; i.e., $\lim \sup _{\lambda \rightarrow \infty} \frac{1}{r} \ln \|a(\lambda)\| \leq-T$,

(b) $\lim \sup _{r \rightarrow \infty} \frac{1}{r} \ln \| a\left(r e^{i \theta)} \| \leq-T \cos \theta\right.$ for all $-\phi<\theta<\phi$.

Lemma 5. Let $f \in L_{\text {loc }}^{1}([0, \infty), X)$ such that $\hat{f}(\lambda)$ exists for $\lambda>\omega>0$. Then $\hat{f} \in\{f\}_{i}$ for $i=1,2,3$. Moreover

(a) $\hat{f}$ is of minimal exponential type on the half plane $\operatorname{Re} \lambda>\omega+\varepsilon$, for any $\varepsilon>0$ and

(b) $a(\lambda):=\int_{T}^{\infty} e^{-\lambda t} f(t) d t$ is of minimal exponential type on the half plane $\operatorname{Re} \lambda>\omega+\varepsilon$ and $a \approx_{T} 0$ for any $T>0$.

Remark 6. Let $f \in L_{l o c}^{1}([0, \infty), X)$. Then the finite Laplace transform

$$
\hat{f}_{T}(\lambda):=\int_{0}^{\infty} e^{-\lambda t} f(t) \chi_{[0, T]}(t) d t=\int_{0}^{T} e^{-\lambda t} f(t) d t
$$

is bounded on the half plane $\{\lambda: R e \lambda>0\}$. In particular $\hat{f}_{T}(\lambda) \in\{f\}_{i}^{T}$ for $i=1,2,3$.

The main benefit of these definitions is that all operational properties of the Laplace transform extend to the asymptotic Laplace transform. Clearly, $f \rightarrow\{f\}_{i}$ is linear: i.e., $\{(f+g)\}_{i}=\{f\}_{i}+\{g\}_{i}$ and $\{c f\}_{i}=c\{f\}_{i}$ for $f, g \in$ $L_{\text {loc }}^{1}([0, \infty), X), c \in \mathbb{C}$ and $i=1,2,3$. Also notice that $\approx_{T}$ defines an equivalence relation on $O(\Sigma, X)$. Furthermore, if $r \in O(\Sigma, X)$ and $q \in O(\Sigma, C)$ with $r \approx_{T} 0$ and $q \approx_{S} 0$, then $q r \approx_{T+S} 0$. The same holds for $O(S, X)$ and $\mathcal{A}(\Sigma, X)$.

Theorem 7 (Existence and Uniqueness). ${ }^{1}$ Let $f, g \in L_{l o c}^{1}([0, \infty) ; X)$ and $0<T \leq \infty$. Then for $i=1,2,3$

(a) $\emptyset \neq\{f\}_{i} \subset\{f\}_{i}^{T}$

(b) if $\hat{f}$ exists, then $\hat{f} \in\{f\}_{i}$;

(c) $\{f\}_{i}^{T} \cap\{g\}_{i}^{T} \neq \emptyset$ if and only if $f=g$ a.e. on $[0, T]$.

\footnotetext{
${ }^{1}$ This theorem is essentially due to Vignaux [11] who extended Ritt's Theorem about asymptotic series to asymptotic integrals; see, e.g., R. Remmert [10], 9.6 .4 and also J.C. Vignaux and M. Cotlar [12], L. Berg [2]. In the present form, the result was proved for \{\}$_{1}$ by G. Lumer and F. Neubrander in [7].
} 
The usefulness of the Laplace transform in applications to differential and integral equations is due to the fact that it maps differentiation, integration, and more generally, convolution onto multiplication. These crucial operational properties extend to the asymptotic Laplace transform as well. Recall that if $f \in L_{l o c}^{1}([0, \infty), X)$ and,$g \in L_{l o c}^{1}([0, \infty), \mathbb{R})$ then

$$
f * g: t \rightarrow \int_{0}^{t} f(t-s) g(s) d s \quad(t \geq 0)
$$

denotes the convolution of $f$ and $g$. For all scalar, locally Lebesgue integrable functions functions $g, h$ and locally Bochner integrable functions $f$ the convolution $f * g$ is in $L_{l o c}^{1}[0, \infty), f * g=g * f$, and $(g * h) * f=g *(h * f){ }^{2}$ To simplify notation we define $\{f\}_{i}^{\infty}:=\{f\}$.

Proposition 8. Let $0<T \leq \infty, i \in\{1,2,3\}$, and $f \in L_{l o c}^{1}([0, \infty) ; X)$. If $f$ is absolutely continuous and $f^{\prime}$ exists a.e., then

$$
\left\{f^{\prime}\right\}_{i}^{T}=\lambda\{f\}_{i}^{T}-f(0) .
$$

If $g \in L_{l o c}^{1}([0, \infty) ; \mathbb{C})$, then

$$
\{f * g\}_{i}^{T}=\{f\}_{i}^{T} \cdot\{g\}_{i}^{T} .
$$

Proposition 9. Let $f \in L_{l o c}^{1}([0, \infty), X)$ and $i \in\{2,3\}$. Then

$$
\{-t f\}_{i}=\{f\}_{i}^{\prime} .
$$

We turn now to the question about the characteristic properties of an equivalence class $\{f\}_{i}$. We will prove below that if one of the members of the asymptotic class $\{f\}$ has an asymptotic expansion in terms of $\frac{1}{\lambda}$ as $\lambda \rightarrow \infty$ then all members of the asymptotic Laplace transform have the same asymptotic expansion. The investigation of this topic is what follows next.

Recall that $f(x)=O(g(x))$ as $x \rightarrow \infty$ if there exists $a>0$ such that $\|f(x)\| \leq M\|g(x)\|$ for $x \in[a, \infty)$. Also, $f(x)=o(g(x))$ as $x \rightarrow \infty$ if for each $\varepsilon>0$ there exists $a_{\varepsilon}$ such that $\|f(x)\| \leq \varepsilon\|g(x)\|$ for all $x \in\left[a_{\varepsilon}, \infty\right)$. The following properties of the order symbols hold.

1. If $c_{1}, c_{2}$ are constants, then $c_{1} O(g)+c_{2} O(g)=O(g)$.

2. If $c_{1}, c_{2}$ are constants, then $c_{1} o(g)+c_{2} o(g)=o(g)$.

3. $O(O(g))=O(g)$.

\footnotetext{
${ }^{2}$ For a proof of the scalar case, see N. Dunford and J. Schwartz [6], VIII.1.24, 633-636.
} 
4. $O(o(g))=o(O(g))=o(o(g))=o(g)$.

5. $O(f) O(g)=O(f g)$.

6. $O(f) o(g)=o(f g)$.

A finite or infinite sequence of functions $\left\{\phi_{n}(x)\right\}_{n \in \mathbb{N}}$ defined on an interval $(a, \infty)$ is called an asymptotic sequence as $x \rightarrow \infty$ if the following two conditions are satisfied:

(1) $\phi_{n}(x) \neq 0$, for $n=1,2 \ldots$

(2) $\phi_{n+1}(x)=o\left(\phi_{n}(x)\right)$, as $x \rightarrow \infty$.

Obvious examples of asymptotic sequences are given by $\phi_{n}(x)=\frac{1}{x}$ or $\phi_{n}(x)=$ $e^{-n x}$. We say that a function $f(x)$ has an asymptotic development to $N$ terms with respect to the asymptotic sequence $\left\{\phi_{n}(x)\right\}_{n \in \mathbb{N}}$ if there exists constants $c_{1}, c_{2}, \ldots, c_{N}$ such that

$$
f(x)=c_{1} \phi_{1}(x)+\ldots+c_{N} \phi_{N}(x)+o\left(\phi_{N}(x)\right) \text { as } x \rightarrow \infty .
$$

In the case that $f(x)$ has an asymptotic development to $N$ terms for any $N \in$ $\mathbb{N}$, we say that $f(x)$ has an asymptotic expansion in terms of the sequence $\left\{\phi_{n}(x)\right\}_{n \in \mathbb{N}}$ and write

$$
f(x) \sim \sum_{n=1}^{\infty} c_{n} \phi_{n}(x) \text { as } x \rightarrow \infty .
$$

\section{Main Results}

The following theorem is essentially due to G. Lumer (unpublished).

Theorem 10. Let $f:[0, \infty) \rightarrow X$ extend to an entire analytic function $f(z)=a_{0}+a_{1} z+a_{2} z^{2}+\ldots$. Then each $r \in\{f\}_{i}$ admits the uniquely determined asymptotic expansion at $\infty$ in $\frac{1}{\lambda^{n}}$

$$
\{f\}(\lambda) \sim \frac{a_{0}}{\lambda}+\frac{a_{1}}{\lambda^{2}}+\frac{2 ! a_{2}}{\lambda^{3}}+\ldots
$$

Proof. We must show that there exists a sequence of vectors $\left(a_{n}\right)_{n \in \mathbb{N}}, a_{n} \in X$, such that for any $u \in\{f\}_{i}$ and any $n \geq 0$,

$$
u(\lambda)=\frac{a_{0}}{\lambda}+\ldots+\frac{n ! a_{n}}{\lambda^{n+1}}+o\left(\frac{1}{\lambda^{n+1}}\right)
$$


as $\lambda \rightarrow \infty$. We do this in several steps.

a) Let $T>0$, and $n \in \mathbb{N}$ be fixed. Then $\hat{f}_{T}(\lambda) \approx_{T} u(\lambda)$, where

$$
\hat{f}_{T}(\lambda)=\int_{0}^{T} e^{-\lambda t} f(t) d t=I_{1}+I_{2}
$$

with

$$
\begin{aligned}
& I_{1}:=\int_{0}^{T} e^{-\lambda t}\left(a_{0}+a_{1} t+a_{2} t^{2}+\ldots+a_{n} t^{n}\right) d t \\
& I_{2}:=\int_{0}^{T} e^{-\lambda t}\left(a_{n+1} t^{n+1}+\ldots\right) d t
\end{aligned}
$$

Since $f(z)$ is entire we have that $\sqrt[i]{\left\|a_{j}\right\|} \rightarrow 0$; i.e for any $\epsilon>0$ there exists $n_{\epsilon}>n$ such that

$$
\left\|a_{j}\right\| \leq(\epsilon)^{j}
$$

for $j \geq n_{\epsilon}$. Let $0<\alpha<1$ and choose $\epsilon>0$ such that $T \epsilon \leq \alpha$. Write $I_{2}=I_{2}^{* *}+I_{2}^{*}$, where

$$
I_{2}^{*}:=\int_{0}^{T} e^{-\lambda t} \sum_{m \geq n_{\epsilon}} a_{m} t^{m} d t
$$

and

$$
I_{2}^{* *}:=\int_{0}^{T} e^{-\lambda t} \sum_{m=n+1}^{n_{\epsilon}-1} a_{m} t^{m} d t .
$$

Let $\sup _{t \in[0, T]}\left\|\sum_{m=n+1}^{n_{\varepsilon}-1} a_{m} t^{m-n-1}\right\|:=M\left(n_{\varepsilon}, T\right)$. Then

$$
\left\|I_{2}^{* *}\right\| \leq M\left(n_{\epsilon}, T\right) \int_{0}^{T} e^{-\lambda t} t^{n+1} d t \leq \frac{M\left(n_{\varepsilon}, T\right)(n+1) !}{\lambda^{n+2}} .
$$

In particular, $\left\|I_{2}^{* *}\right\|=o\left(\frac{1}{\lambda^{n+2}}\right)$ as $\lambda \rightarrow \infty$. For $I_{2}^{*}$ we have

$$
\begin{aligned}
\left\|\sum_{m \geq n_{\epsilon}} a_{m} t^{m}\right\| & \leq t^{n_{\epsilon}}\left(\epsilon^{n_{\epsilon}}+\epsilon^{n_{\epsilon}+1} t+\epsilon^{n_{\epsilon}+2} t^{2}+\ldots\right) \\
& \leq \frac{\alpha^{n_{\epsilon}}}{T^{n_{\epsilon}}} t^{n_{\epsilon}}\left(1+\alpha+\alpha^{2}+\ldots\right) \\
& =\frac{\alpha^{n_{\epsilon}}}{T^{n_{\epsilon}}} \frac{1}{1-\alpha} t^{n_{\epsilon}} .
\end{aligned}
$$


Thus,

$$
\begin{aligned}
\left\|I_{2}^{*}\right\| & \leq \int_{0}^{T} e^{-\lambda t}\left\|\sum_{m \geq n_{\epsilon}} a_{m} t^{m}\right\| d t \\
& \leq \frac{\alpha^{n_{\epsilon}}}{T^{n_{\epsilon}}} \frac{1}{1-\alpha} \frac{\left(n_{\epsilon}\right) !}{\lambda^{n_{\epsilon}+1}} \\
& \leq \frac{M^{*}}{\lambda^{n+2}}
\end{aligned}
$$

if $\lambda>1$ and $M:=\frac{\alpha^{n_{\epsilon}}}{T^{n_{\epsilon}}} \frac{\left(n_{\epsilon}\right) !}{1-\alpha}$. In particular,

$$
\left\|I_{2}\right\|=O\left(\frac{1}{\lambda^{n+2}}\right)=o\left(\frac{1}{\lambda^{n+1}}\right)
$$

as $\lambda \rightarrow \infty$.

b) To estimate $I_{1}=\int_{0}^{T} e^{-\lambda t}\left(a_{0}+a_{1} t+a_{2} t^{2}+\ldots+a_{n} t^{n}\right) d t$ define

$$
\begin{aligned}
E_{1}(\lambda): & =\int_{0}^{\infty} e^{-\lambda t}\left(a_{0}+\ldots+a_{n} t^{n}\right) d t-I_{1} \\
& =\int_{T}^{\infty} e^{-\lambda t}\left(a_{0}+a_{1} t+\ldots+a_{n} t^{n}\right) d t .
\end{aligned}
$$

Let $M_{1}:=\sup _{t \in[T, \infty)}\left\|\frac{a_{0}}{t^{n}}+\ldots+a_{n}\right\|$. Then

$$
\begin{aligned}
\left\|E_{1}(\lambda)\right\| & \leq \int_{T}^{\infty} e^{-\lambda t} t^{n}\left\|\frac{a_{0}}{t^{n}}+\frac{a_{1}}{t^{n-1}}+\ldots+a_{n}\right\| d t \\
& \leq M_{1} \int_{T}^{\infty} e^{-\lambda t} t^{n} d t \\
& \leq M_{1} e^{-\lambda T}\left(\frac{T^{n}}{\lambda}+\frac{n T^{n-1}}{\lambda^{2}}+\ldots+\frac{n !}{\lambda^{n+1}}\right) \\
& \leq M_{2}(n, T) e^{-\lambda T}
\end{aligned}
$$

where $M_{2}(n, T)=\sup _{\lambda \geq 1}\left|\frac{T^{n}}{\lambda}+\frac{n T^{n-1}}{\lambda^{2}}+\ldots+\frac{n !}{\lambda^{n+1}}\right|=T^{n}+n T^{n-1}+\ldots+n !$

To finish the proof, let $a(\lambda):=u(\lambda)-\hat{f_{T}}(\lambda) \approx_{t} 0$. In particular, for all $\mu>0$ there exists $M_{\mu}>0$ such that $\|a(\lambda)\| \leq M_{\mu} e^{-\lambda(T+\mu)}$. This implies that $\|a(\lambda)\|=O\left(e^{-\lambda(T+\mu)}\right)$ for all $\mu>0$. Therefore,

$$
\left\|u(\lambda)-\left(\frac{a_{0}}{\lambda}+\frac{a_{1}}{\lambda^{2}}+\ldots+\frac{n ! a_{n}}{\lambda^{n+1}}\right)\right\|
$$




$$
\begin{aligned}
& \leq\left\|u(\lambda)-\hat{f_{T}}(\lambda)\right\|+\left\|I_{1}(\lambda)+I_{2}(\lambda)-\left(\frac{a_{0}}{\lambda}+\frac{a_{1}}{\lambda^{2}}+\ldots+\frac{n ! a_{n}}{\lambda^{n+1}}\right)\right\| \\
& \leq\left\|u(\lambda)-\hat{f_{T}}(\lambda)\right\|+\left\|I_{2}(\lambda)\right\|+\left\|E_{1}(\lambda)\right\| \\
& \leq O\left(e^{-\lambda(T+\mu)}\right)+o\left(\frac{1}{\lambda^{n+1}}\right)+O\left(e^{-\lambda T}\right) \\
& =o\left(\frac{1}{\lambda^{n+1}}\right)
\end{aligned}
$$

for any $\mu>0$. Hence 2 holds so the theorem is proved.

This theorem has inspired us to prove the following result, which in essence states that if one of the functions in the elements of the equivalence class of $\{f\}$ has an asymptotic expansion in terms of $\frac{1}{\lambda}$ as $\lambda \rightarrow \infty$, then any other element of the equivalence class has the same asymptotic expansion.

Proposition 11. Let $r_{1}, r_{2} \in\{f\}_{i}$ for some $f \in L_{l o c}^{1}([0, \infty), X)$ be such that $r_{1}$ has an asymptotic expansion in terms of $\frac{1}{\lambda}$ as $\lambda \rightarrow \infty$. Then $r_{2}$ has the same asymptotic expansion in terms of $\frac{1}{\lambda}$ as $\lambda \rightarrow \infty$.

Proof. Suppose that $r_{1}, r_{2} \in\{f\}_{i}$ and

$$
r_{1}(\lambda)=a_{0}+\frac{a_{1}}{\lambda}+\ldots+\frac{a_{n}}{\lambda^{n}}+o\left(\frac{1}{\lambda^{n+1}}\right) .
$$

from the definition of the asymptotic Laplace transform we have that for any $T>0$

$$
r_{i}(\lambda)-\int_{0}^{T} e^{-\lambda t} f(t) d t \approx_{T} 0
$$

for $i=1,2$. We need to show that

$$
r_{2}(\lambda)-a_{0}-\frac{a_{1}}{\lambda}-\ldots-\frac{a_{n}}{\lambda^{n}}=o\left(\frac{1}{\lambda^{n+1}}\right)
$$

as $\lambda \rightarrow \infty$. Since

$$
\begin{aligned}
& \left\|r_{2}(\lambda)-a_{0}-\frac{a_{1}}{\lambda}-\ldots-\frac{a_{n}}{\lambda^{n}}\right\|= \\
& =\left\|r_{2}(\lambda)-\int_{0}^{T} e^{-\lambda t} f(t) d t+\int_{0}^{T} e^{-\lambda t} f(t) d t-r_{1}(\lambda)+r_{1}(\lambda)-a_{0}-\ldots-\frac{a_{n}}{\lambda^{n}}\right\| \\
& \leq\left\|r_{2}(\lambda)-\int_{0}^{T} e^{-\lambda t} f(t) d t\right\|+\left\|\int_{0}^{T} e^{-\lambda t} f(t) d t-r_{1}(\lambda)\right\| \\
& \quad+\left\|r_{1}(\lambda)-a_{0}-\ldots-\frac{a_{n}}{\lambda^{n}}\right\|
\end{aligned}
$$




$$
\begin{aligned}
& \leq e^{-\lambda T}+e^{-\lambda T}+o\left(\frac{1}{\lambda^{n+1}}\right) \\
& =o\left(\frac{1}{\lambda^{n+1}}\right)
\end{aligned}
$$

we have that

$$
r_{2}(\lambda)=a_{0}+\frac{a_{1}}{\lambda}+\ldots+\frac{a_{n}}{\lambda^{n}}+o\left(\frac{1}{\lambda^{n+1}}\right)
$$

and the proof is complete.

Recall that $f(x)=O(g(x))$ as $x \rightarrow 0^{+}$if there exists $a>0$ such that $\|f(x)\| \leq M\|g(x)\|$ for $x \in(0, a)$. Also, $f(x)=o(g(x))$ as $x \rightarrow 0$ if for each $\varepsilon>0$ there exists $a_{\varepsilon}$ such that $\|f(x)\| \leq \varepsilon\|g(x)\|$ for all $x \in\left(0, a_{\varepsilon}\right)$ Next we prove a generalization of Watson's Lemma for the asymptotic Laplace transform.

Theorem 12 (Watson's Lemma). Suppose that $f \in L_{l o c}^{1}([0, \infty), X)$ has an asymptotic expansion in terms of $\left\{t^{n}\right\}_{n \in \mathbb{N}}$ as $t \rightarrow 0^{+}$; i.e., suppose that

$$
f(t) \sim \sum_{n=0}^{\infty} c_{n} t^{n}
$$

Then, for any $u \in\{f\}_{i}$ we have that

$$
u(\lambda) \sim \sum_{n=0}^{\infty} c_{n} \frac{n !}{\lambda^{n+1}} \text { as } \lambda \rightarrow \infty
$$

Proof. We need to show that

$$
\left\|u(\lambda)-\sum_{n=0}^{N} c_{n} \frac{n !}{\lambda^{n+1}}\right\|=o\left(\frac{1}{\lambda^{N+1}}\right) \text { as } \lambda \rightarrow \infty
$$

for any $N>0$. Let $T>0$. Since $\sum_{n=0}^{N} c_{n} \frac{n !}{\lambda^{n+1}}=\int_{0}^{\infty} \sum_{n=0}^{N} e^{-\lambda t} c_{n} t^{n} d t$ we have that

$$
\begin{aligned}
\left\|u(\lambda)-\sum_{n=0}^{N} c_{n} \frac{n !}{\lambda^{n+1}}\right\| & =\left\|u(\lambda)-\sum_{n=0}^{N} \int_{0}^{\infty} e^{-\lambda t} c_{n} t^{n} d t\right\| \\
& =\left\|u(\lambda)-\int_{0}^{T} \sum_{n=0}^{N} c_{n} t^{n} e^{-\lambda t} d t-\int_{T}^{\infty} \sum_{n=0}^{N} c_{n} t^{n} e^{-\lambda t} d t\right\| \\
& \leq\left\|u(\lambda)-\int_{0}^{T} \sum_{n=0}^{N} c_{n} t^{n} e^{-\lambda t} d t\right\|+\left\|\int_{T}^{\infty} \sum_{n=0}^{N} c_{n} t^{n} e^{-\lambda t} d t\right\|
\end{aligned}
$$




$$
=I_{1}+I_{2}
$$

Now

$$
\begin{aligned}
I_{1} & =\left\|u(\lambda)-\int_{0}^{T} e^{-\lambda t} f(t) d t+\int_{0}^{T} e^{-\lambda t} f(t) d t-\int_{0}^{T} \sum_{n=0}^{N} c_{n} t^{n} e^{-\lambda t} d t\right\| \\
& \leq\left\|u(\lambda)-\int_{0}^{T} e^{-\lambda t} f(t) d t\right\|+\left\|\int_{0}^{T} e^{-\lambda t}\left(f(t)-\sum_{n=0}^{N} c_{n} t^{n}\right) d t\right\| \\
& \leq\|a(\lambda)\|+\left\|\int_{0}^{T} e^{-\lambda t}\left(f(t)-\sum_{n=0}^{N} c_{n} t^{n}\right) d t\right\|
\end{aligned}
$$

From the definition of the asymptotic Laplace transform it follows that for any $\varepsilon>0\|a(\lambda)\| \leq e^{-\lambda(T-\varepsilon)}$ for sufficiently large $\lambda$ and from the fact that $f(t) \sim \sum_{n=0}^{\infty} c_{n} t^{n}$ and by using Theorem 1.16 pg $29[3]$ for the function $(f(t)-$ $\left.\sum_{n=0}^{N} c_{n} t^{n}\right) \chi_{[r, T]}$ it follows that

$$
\begin{aligned}
& \left\|\int_{0}^{T} e^{-\lambda t}\left(f(t)-\sum_{n=0}^{N} c_{n} t^{n}\right) d t\right\| \\
\leq & \int_{0}^{r} e^{-\lambda t}\left\|f(t)-\sum_{n=0}^{N} c_{n} t^{n}\right\| d t+\left\|\int_{r}^{T} e^{-\lambda t}\left(f(t)-\sum_{n=0}^{N} c_{n} t^{n}\right) d t\right\| \\
\leq & \int_{0}^{r} e^{-\lambda t} c_{N} t^{N} d t+\left\|\int_{r}^{T} e^{-\lambda t}\left(f(t)-\sum_{n=0}^{N} c_{n} t^{n}\right) \chi_{[r, T]} d t\right\| \\
\leq & \int_{0}^{\infty} e^{-\lambda t} c_{N} t^{N} d t+M e^{-\lambda r} d t \\
= & c_{N} \frac{(N) !}{\lambda^{N+1}}+M e^{-\lambda r} \\
= & o\left(\frac{1}{\lambda^{N+2}}\right) .
\end{aligned}
$$

It remains to be shown that $I_{2}=o\left(\frac{1}{\lambda^{n+1}}\right)$.

$$
\begin{aligned}
I_{2} & =\left\|\int_{T}^{\infty} \sum_{n=0}^{N} c_{n} t^{n} e^{-\lambda t} d t\right\| \\
& \leq M_{1} \int_{T}^{\infty} e^{-\lambda t} t^{n} d t
\end{aligned}
$$




$$
\begin{aligned}
& \leq M_{1} e^{-\lambda T}\left(\frac{T^{n}}{\lambda}+\frac{n T^{n-1}}{\lambda^{2}}+\ldots+\frac{n !}{\lambda^{n+1}}\right) \\
& \leq M_{2}(n, T) e^{-\lambda T},
\end{aligned}
$$

where $M_{2}(n, T)=\sup _{\lambda \geq 1}\left|\frac{T^{n}}{\lambda}+\frac{n T^{n-1}}{\lambda^{2}}+\ldots+\frac{n !}{\lambda^{n+1}}\right|=T^{n}+n T^{n-1}+\ldots+n !$ In particular $I_{2}=O\left(e^{-\lambda T}\right)$ and therefore $I_{2}=o\left(\frac{1}{\lambda^{n+1}}\right)$. Thus

$$
\begin{aligned}
\left\|u(\lambda)-\sum_{n=0}^{N} c_{n} \frac{n !}{\lambda^{n+1}}\right\| & \leq o\left(\frac{1}{\lambda^{N+1}}\right)+O\left(e^{-\lambda T}\right) \\
& =o\left(\frac{1}{\lambda^{N+2}}\right)
\end{aligned}
$$

for any $N>0$ and the proof is complete.

\section{References}

[1] W. Arendt, C.J.K. Batty, M. Hieber and F. Neubrander, Vector-Valued Laplace Transforms and Cauchy Problems, Monographs in Mathematics, Birkhäuser Verlag, 2001.

[2] L. Berg, Asymptotiche Auffassung der Operatorenrechnung, Studia Math. 21, 1962, 215-229.

[3] C. Mihai, Asymptotic Laplace Transforms, Dissertation, Louisiana State University, 2004.

[4] M. Deakin, Laplace transform for superexponential functions, J. Austral. Math. Soc. Ser., B 36, 1994, 17-25.

[5] M. Ditkin, On the theory of operational calculus, Dokl. Akad. Nauk SSSR, 123, 1958, 395-396.

[6] N. Dunford and J. Schwartz, Linear Operators, Vol. I, Interscience, 1958.

[7] G. Lumer and F. Neubrander, Asymptotic Laplace Transforms and evolution equations, Advances in Partial Differential Equations, Vol. 16, WileyVCH, 1999.

[8] G. Lumer and F. Neubrander, The Asymptotic Laplace Transforms: New Results and Relation to Komatsu's Laplace Transform of Hyperfunctions, Partial Differential Equations on Multistructures, Lecture Notes Pure and Applied Mathematics 219, Marcel Dekker, 2001. 
[9] Y.I. Lyubich, The classical and local Laplace transformation in an abstract Cauchy problem. Usepi Math. Nauk, 21, 1966, 3-51.

[10] R. Remmert, Theory of Complex Functions, Graduate Texts in Mathematics 122, Springer-Verlag, New York, 1991.

[11] J.C. Vignaux, Sugli integrali di Laplace asintotici., Atti Accad. naz. Lincei Rend., 1939, 345-400.

[12] J.C. Vignaux and M. Cotlar, Asymptotic Laplace-Stieltjes integrals, Univ. Nac. La Plata. Publ. Fac. Ci. Fisicomat., (2) 3(14), 1944, 345-400. 
